\title{
miR520c blocks EMT progression of human breast cancer cells by repressing STAT3
}

\author{
NIAN WANG, LAN WEI, YUNXIU HUANG, YANG WU, MIN SU, XUELI PANG, \\ FEIHU JI, CHANGLI ZHONG, TINGMEI CHEN and BING LI
}

Chongqing Medical University, Yuzhong, Chongqing 400016, P.R. China

Received August 1, 2016; Accepted December 27, 2016

DOI: $10.3892 / o r .2017 .5393$

\begin{abstract}
Breast cancer is one of the most malignant diseases world-wide and it ranks the first among female cancers. Masses of intrinsic and extrinsic factors, especially the inflammatory factors can lead to breast cancer. Aberrant activation and accumulation of key molecules can lead to inflammation associated carcinogenesis. The signal transducers and activators of transcription 3 (STAT3) is one of them. Therefore, to evaluate the novel molecular mechanisms, STAT3 has become our focus for breast cancer targeted therapy. At present, many tumor suppressing microRNAs have been validated, and are the highlights in research on microRNAs. Thus, we predicted microRNAs which could putatively regulate STAT3 through databases and selected six to screen with Dual-luciferase assay. The result hinted that miR520c could bind with STAT3 3'UTR. We mutated the seed sequence of miR520c on STAT3 3'UTR, which illustrated a reverse effect compared with wild-type of STAT3 3'UTR. Subsequently, STAT3, p-STAT3 and miR520c were assessed in three different grades of breast cancer cells, with the degree of malignancy, we found an escalating trend of STAT3 and p-STAT3, on the contrary, a downward trend of miR520c. We observed STAT3 was deactivated by miR520c. Epithelial to mesenchymal transition (EMT) is a fatal transfer of cancer progression. To find out whether the downregulation of STAT3 can repress breast cancer motility and invasion ability, we detected EMT markers. The result implied a suppression effect on EMT. We overexpressed STAT3 to conduct rescue experiments, the result showed a recovery of STAT3 and EMT characteristics. Cell motility and invasion property were regained as well. In the study, we elucidated miR520c could inhibit breast cancer EMT by targeting STAT3. It can enrich the mechanism of breast cancer and may lay the foundation for breast cancer targeted treatment.
\end{abstract}

Correspondence to: Professor Bing Li, Chongqing Medical University, 1 Road Yixueyuan, Yuzhong, Chongqing 400016, P.R. China

E-mail: 1390658110@qq.com

Key words: miR520c, STAT3, breast cancer, epithelial to mesenchymal transition, targeted therapy

\section{Introduction}

According to the statistics of WHO, breast cancer has become the number one killer among female cancers. Though, the cure percentage of early stage breast cancer has reached $80-90 \%$, the late stage of this disease is still indisputable (1). Accordingly, to search for more sensitive diagnostic indicators and therapeutic methods is of great importance. A recent molecular testing of breast cancer has shown, during the carcinogenesis and progression of cancer, a number of molecules and signaling pathways participate having respective functions. Thus breast cancer can be divided into four subtypes depending on the molecular features: Luminal A, Luminal B, HER $2^{+}$and basal like (2). Cancer cells exist in a complicated microenvironment, the surrounding cells crosstalk with malignant cancer cells and confer to the progression of cancer (3). Various cytokines and regulators are involved. Interleukin (IL)-24 transforms the tumor microenvironment in colon cancer (4). $\beta$-catenin, PPAR- $\gamma$, and FGFR3 pathways are activated and drives non-T cell-inflamed tumor microenvironment in urothelial bladder cancer (5). Cytokines produced by stromal cells are connected with tumor grades and survival percentage. As reported, IL-1 $\beta$ and IL-17 are positively associated with histological grade; IFN $\beta$ expressed higher, and NF- $\mathrm{kB}$ lower in HER-2-positive tumors; and IL-6 was shown with a higher global expression in node-negative tumors (6). Recent data suggests that immunity associated molecules of tumor microenvironment play an important role in breast cancer malignancy (7), STATs family is one of them and has been studied in recent years as vital molecules in inflammation induced cancers.

Signal transducers and activators of transcription (STAT) is a class of cytoplasmic and unclear signaling pathway molecules. The activation of STAT3 is mostly conducted by JAK family. STAT3 is the crucial member of the STAT family. It medicates transcription of several kinds of cytokines and growth factors (8). As is reported, STAT3 is continuously activated in diverse human cancers. It also enhances transcription of oncogenes, and inhibits cell apoptosis in cancers. STAT3 especially plays a core role in inflammation induced cancers, IL6-JAK-STAT3 is the key signaling pathway of carcinogenesis and epigenetic transformation (9). Besides, some extrinsic carcinogenic factors including sunlight, pathogens, chemical cancerogens can also active STAT3 (10). High percentage of 
activated STAT3 has been found in certain cancers, such as thyroid cancer, colorectal cancer, liver cancer, lung cancer, breast cancer, and cancer associated microenvironments (11). Thus, to find a new mechanism that can specifically inhibit STAT3 would be of great therapeutic value.

MicroRNAs are a class of 19-25 nt short non-coding RNAs, which exist in many physiological and pathologic processes $(12,13)$. Mature miRNAs form RNA-induced silencing complex (miRISC) to conversely complement with 3'UTR of the target mRNA, which results in silence or merely degradation of a specific mRNA (14). It has been reported, nearly $30 \%$ of human genes are regulated by microRNAs. Genes of microRNAs surrounded by aberrantly histidine modified CpG island can be named as onco-microRNAs (15), which are always upregulated. MicroRNA expresses abnormally in most of human diseases, particularly in cancers. At present, microRNA expression profiles have been applied for diagnosing and classifying human cancers $(16,17)$.

MicroRNAs have a vital role in the progress of diseases. A microRNA can have completely opposite functions depending on the tissue and the target gene $(18,19)$. MicroRNA-494 promotes cervical cancer proliferation by regulating PTEN (20), but it represses the expression of HOXA10 to inhibit cell proliferation in oral cancer (21).

Some microRNAs are correlated with cancer drug resistance, miR519a has been reported to induce tamoxifen resistance and decrease the survival rate in breast cancer (22). miRNA221/222, known as oncomirs, can cause acquired fulvestrant resistance in MCF cell lines (23). Some are tumor suppressors, miR-520c suppresses NF- $\kappa \mathrm{B}$ and TGF- $\beta$ in estrogen receptor negative breast cancer (24).

Herein, we confirmed miR520c was the corresponding small non-coding RNA for binding with STAT3 3'UTR. Then we discovered miR520c and STAT3 expressed abnormally in different degrees of breast cancer cells. Additionally, miR520c negatively regulated the protein synthesis of STAT3 by direct targeting of STAT3 mRNA. Importantly, the degradation of STAT3 generated prevention consequence on EMT, which abolished progression of breast cancer. No research has been reported on the relevance between miR-520c and STAT3 in cancers. Our study presented here, explain the unknown mechanism between the miR-520c and STAT3, and the inhibition effect on breast cancer in EMT.

\section{Materials and methods}

Cell lines and culture conditions. The human breast cancer cell lines MCF-7, SK-BR-3, MDA-MB-231 and the HEK293T cells were obtained from ATCC and maintained in Dulbecco's modified Eagle's medium or RPMI-1640 (Gibco) with 10\% fetal bovine serum (BI) and antibiotics $(100 \mathrm{mg} / \mathrm{ml}$ streptomycin, $100 \mathrm{U} / \mathrm{ml}$ penicillin, Beyotime) cultured in incubator at $37^{\circ} \mathrm{C}$ and in $5 \% \mathrm{CO}_{2}$.

Plasmids construction. The pri-microRNAs and full-length of STAT3 3'UTR were amplified from human genome (extracted from HEK293T cells with Universal Genomic DNA kit, CWBIO CW2298) and then STAT3 3'UTR was constructed into pCDNA3.1-luc (this vector and other empty plasmids were aquired from Professor Qin Zhou, Laboratory of Molecular
Table I. Primers for plasmid construction.

Pimers Sequences

hsa-mir196b

hsa-mir196b

F: atttcaggtcccggattactggggectgtggcttccc

hsa-mir298

$\mathrm{R}$ : caccaccaccggatccaacctaaccetacctgctgtg

hsa-mir298

$\mathrm{F}$ : aatttcaggtcceggacagccetagctgggttcctaat

hsa-mir494

$\mathrm{R}$ : caccaccaccggatcgecttgccattcatcttctgaa

hsa-mir494

F: aatttcaggtcccggattcattgtgaaggettgaagag

hsa-mir495

$\mathrm{R}$ : caccaccaccggatctccttcaaccacagaagcacag

hsa-mir495

F: aatttcaggtcceggagcetctgctcagtgtcagcc

hsa-mir519b

R: caccaccaccggatcaggcctcgccaactgtgcct

hsa-mir519b

F: aatttcaggtcccggaggatttcccttgatgaacaag

hsa-mir520c

$\mathrm{R}$ : caccaccaccggatcaagaggaccgtttgagcctaaa

hsa-mir520c

F: aatttcaggtcccggaggaggattgcccgttgatga

pCDNA3.1-luc-

$\mathrm{R}$ : caccaccaccggatcctacatactagtgcttgggc

STAT3 3'UTR

pCDNA3.1-luc- $\quad$ R: agcectctagactcgatgaatgcagtggccaggaca STAT3 3'UTR

$\mathrm{F}$, forward primer. $\mathrm{R}$, reverse primer.

Nephrology, Chongqing Medical University) namely STAT3 3'UTR wild-type (WT). The STAT3 3'UTR mutation (Mut) was obtained through site mutation PCR (PrimeStar; Takara). hsa-pri-miR196b, hsa-pri-miR298, hsa-pri-miR494, hsa-pri-miR495, hsa-pri-miR519b, hsa-pri-miR517a/519d, hsa-pri-miR520c microRNA overexpression plasmids were constructed into pdsAAV-CB-EGFP. The primer sequences are in Table I.

Luciferase reporter assay. Cells $\left(0.1 \times 10^{5}\right)$ were seeded into 24-well plate per well. In 16-24 h, following with primary microRNAs (500 ng), pCDNA3.1-luc STAT3 3'UTR WT/ Mut (500 ng), pRL-SV40 (10 ng) were co-transfected into each well by Lipofectamine 2000 (Invitrogen). After $24 \mathrm{~h}$, cells were washed with PBS (pH 7.4) and lysed with diluted $5 \mathrm{X}$ lysis buffer on ice for $30 \mathrm{~min}$ the proteins were collected, luciferase and Renilla activity was measured by Dual-Luciferase Reporter assay system (Promega, USA).

Western blotting. Cells were washed with ice-cold PBS (pH 7.4) and cleaved by RIPA (consisting of $50 \mathrm{mM}$ Tris ( $\mathrm{pH} 7.4$ ), $150 \mathrm{mM} \mathrm{NaCl}, 1 \%$ Triton $\mathrm{X}-100,1 \%$ sodium deoxycholate, $0.1 \%$ SDS, sodium orthovanadate, sodium fluoride, EDTA, and leupeptin, Beyotime) pre-added 100 nM PMSF (phenylmethanesulfonyl fluoride, Sigma). Protein concentration was determined with Bradford protein dye reagent (Beyotime). The loading volumes were evaluated for the equal amount of proteins. Lysate was separated by $8 \%$ SDS-PAGE and blotted onto polyvinylidene fluoride (PVDF) membrane. The membrane was blocked with $5 \%$ fat-free milk then incubated with primary antibodies at $4^{\circ} \mathrm{C}$ overnight. Primary antibodies were diluted at ratios of 1:1,000 ( $\beta$-actin, ZSBio TA-09), 1:500 (STAT3, Boister 1621), 1:1,000 (p-STAT3 (Tyr705), 
A

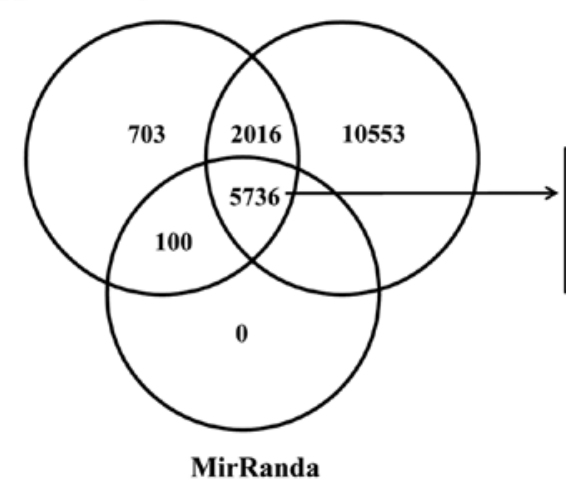

B

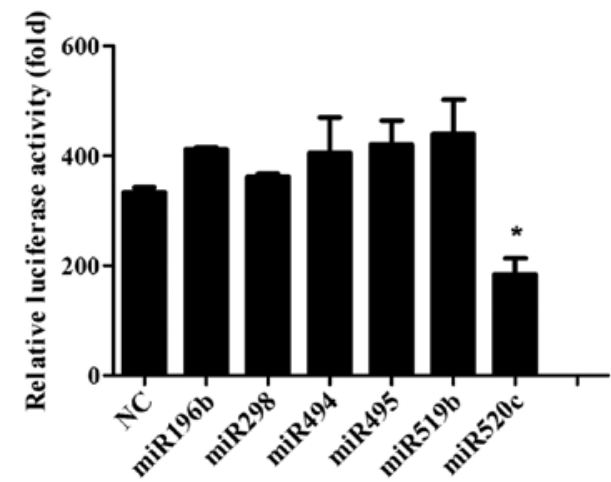

C

Hsa-miR196b, HsamiR298, Hsa-miR494, Hsa-miR495, HsamiR519b, Hsa-miR520c

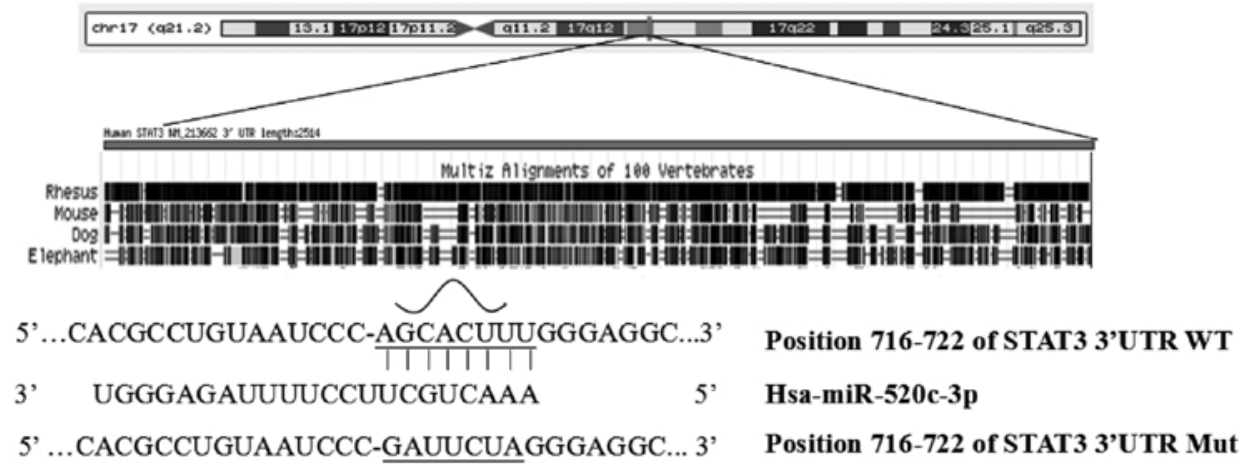

STAT3 3'UTR WT

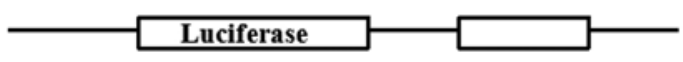

STAT3 3'UTR Mut

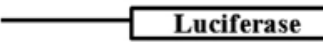

D

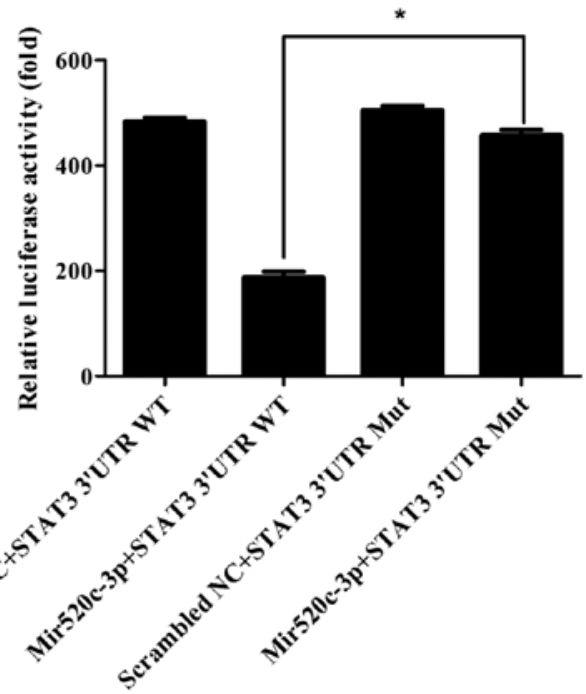

E

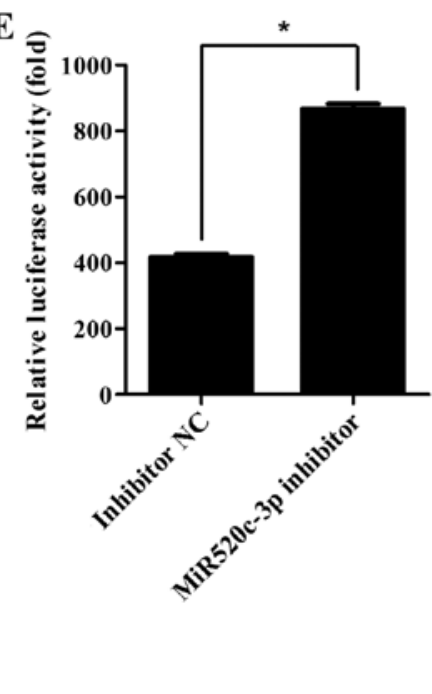

Figure 1. miR520c regulated the 3'UTR of STAT3. (A) Screening results of putative microRNAs among three databases and 6 miRNAs were selected for further determination. (B) 293T cells were co-transfected with pdsAAV/pdsAAV-pri-miRs, STAT3-3'UTR, and pRL-SV40, Dual-luciferase reporter assay was conducted after $24 \mathrm{~h}$. (C) The seed sequences of miR520c on STAT3 3'UTR was analyzed and mutations of the STAT3 3'UTR was contructed with site mutation method. (D) 293T cells were transfected with STAT3-3'UTR-WT/Mut, Dual-luciferase reporter assay was applied after $24 \mathrm{~h}$. (E) miR520c-3p mimics and specific inhibitor were transfected into 293T, Dual-luciferase reporter assay was conducted after $24 \mathrm{~h}$. ${ }^{*} \mathrm{P}<0.05$.

CST \#4113), 1:1,000 (E-cadherin, Bioworld BS1098), 1:1,000 (Vimentin, Bioworld BS1491). Antigen-antibody complex was visualized with immobilon Western chemiluminescent HRP substrate (Millipore, WBKLS0500).
Quantitive RT-PCR. Total RNA was extracted with RNA isoPlus (Takara). Reverse transcription PCR was conducted with random/oligodT and microRNA specific primers (PrimScript RT reagent kit with gDNA Eraser, Takara): miR520c reverse 

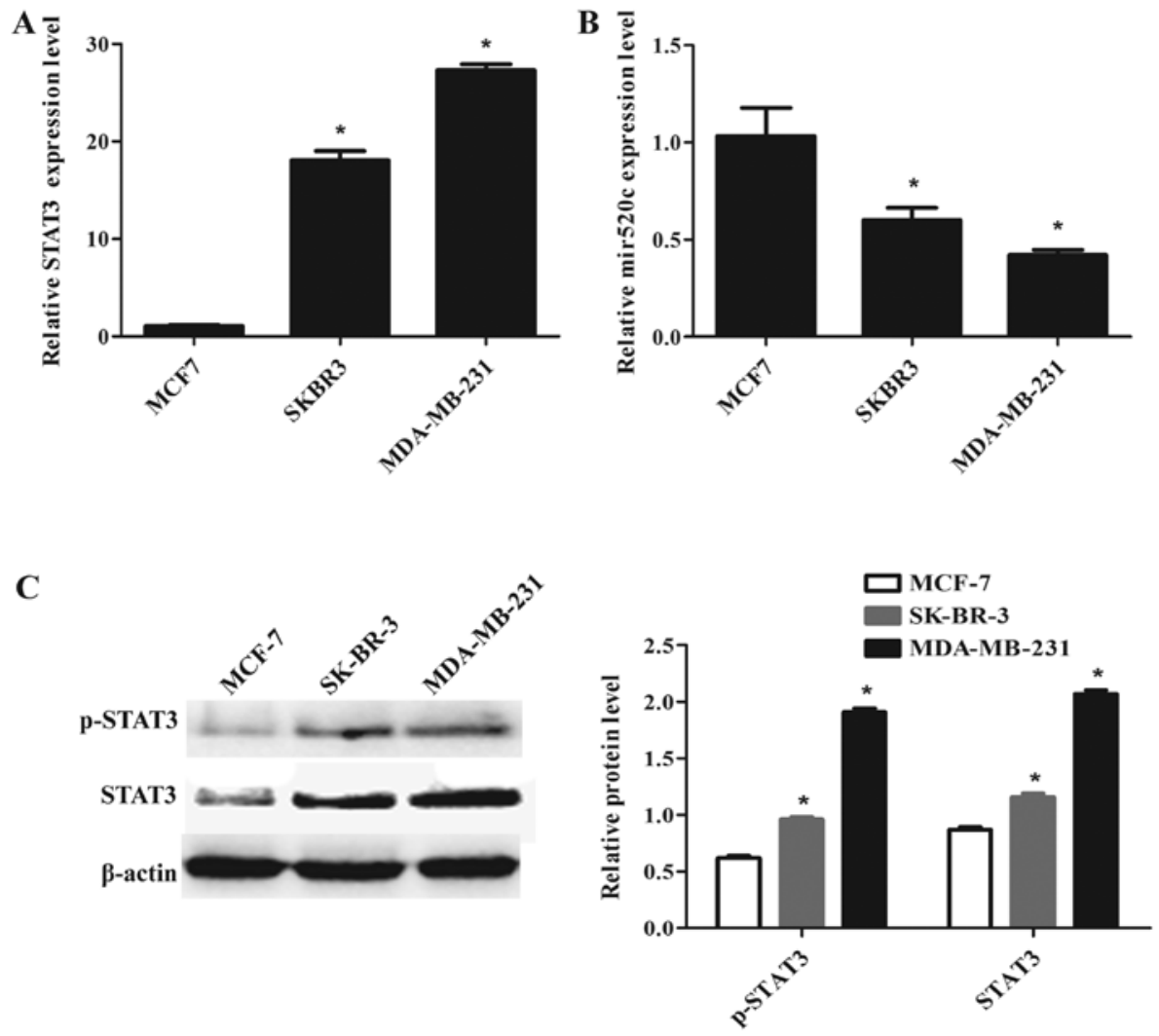

Figure 2. The negative correlation of STAT3 with miR520c. mRNA was extracted from three different grades of cells MCF-7, SK-BR-3 and MDA-MB-231, (A) STAT3 and (B) miR520c expression levels were tested with Q-PCR. (C) Protein was extracted from MCF-7, SK-BR-3, MDA-MB-231, the levels of p-STAT3, STAT3 in three different grades of cells were detected with western blotting. "P<0.05.

oligos 5'-GTCGTATCCAGTGCAGGGTCCGAGGTATTC GCACTGGATACGACCAGAAAGC-3'; hU6 reverse oligos 5'-GTCGTATCCAGTGCAGGGTCCGAGGTATTCGCACT GGATACGACAAAATATGGAAC-3'. Real-time PCR was performed with SYBR Premix Ex Taq II (Takara) and the following primers: miR520c real-time forward, 5'-TGCGGC TCTAGAGGGAAGCGTT-3'; hU6 real-time forward, 5'-TGC GGGTGCTCGCTTCGGCAGC-3'; microRNA universal real-time reverse, 5'-CCAGTGCAGGGTCCGAGGT-3'. The microRNA expession level was normalized by hU6 with $2^{-\Delta \Delta t}$ method.

Cell scratch assay. MCF-7 cells were seeded at the density of $0.25 \times 10^{5}$ cells/well into a 6 -well plate. Forty-eight hours after cells were transfected, a wound was created in each well with a $10-\mu 1$ tip and then washed with PBS three times, followed by imaging at $0 \mathrm{~h}$, and $24 \mathrm{~h}$ under a microscope to record the migration distance at $\mathrm{x} 40$.

Boyden chamber assay. The wells (Millipore, 24-well Millicell) for the 24-well plate were coated with $50 \mu \mathrm{l}$ Matrix gel (Matrigel Matrix, 356234; Corning) at $37^{\circ} \mathrm{C}$ for $30 \mathrm{~min}$. Pre-transfected cells $\left(2 \times 10^{4}\right)$ were seeded into the top of the insert with $1 \%$ fetal bovine serum media, while $10 \%$ fetal bovine serum media was placed in the well below. Then cultured cultured for $24 \mathrm{~h}$, cells still in the top were wiped off with cotton swab, and the migratory cells were stained with crystal violet and captured under the microscope. The invasive cell number in each field was recorded at x200.
Statistical analysis. All statistical analyses were performed by GraphPad Prim 5 software and subjected to Student's t-test (two-tailed, with $\mathrm{P}<0.05$ ) to compare significant differences between the sample means obtained from three independent experiments. Error bars depict SD. Asterisks were used to represent statistical signifiance of P-values in the figures.

\section{Results}

The 3'UTR of STAT3 was targeted by miR520c. STAT3 is consistently activated in several kinds of human cancers and cancer microenvironments. To find which microRNA could directly down regulate STAT3 is of great value in cancer targeted therapy. We predicted microRNAs according to the three authoritative microRNA databases: targetscan, mirwalk and miRanda. Then selected the six top ranking and crossdatabase microRNAs ( $\mathrm{p}<0.01)$ (Fig. 1A). Afterwards, we screened these putative miRNAs by Dual-luciferase reporter assay. Results indicated miR520c downregulated the relative luciferase activity approximately by half compared with the negative control. The other miRNAs were not significantly downregulated (Fig. 1B). So we chose miR520c for further mechanism study. According to database analysis, we found miR520c had only one conservative seed sequence on STAT3 3'UTR (WT) among other species (Fig. 1C). So we constructed the following seed sequence mutated plasmid to verify whether miR520c regulated STAT3 3'UTR through this vital site. Constant with prediction, miR520c had no effect on STAT3 Mut (Fig. 1D). Concordantly, relative luciferase activity 

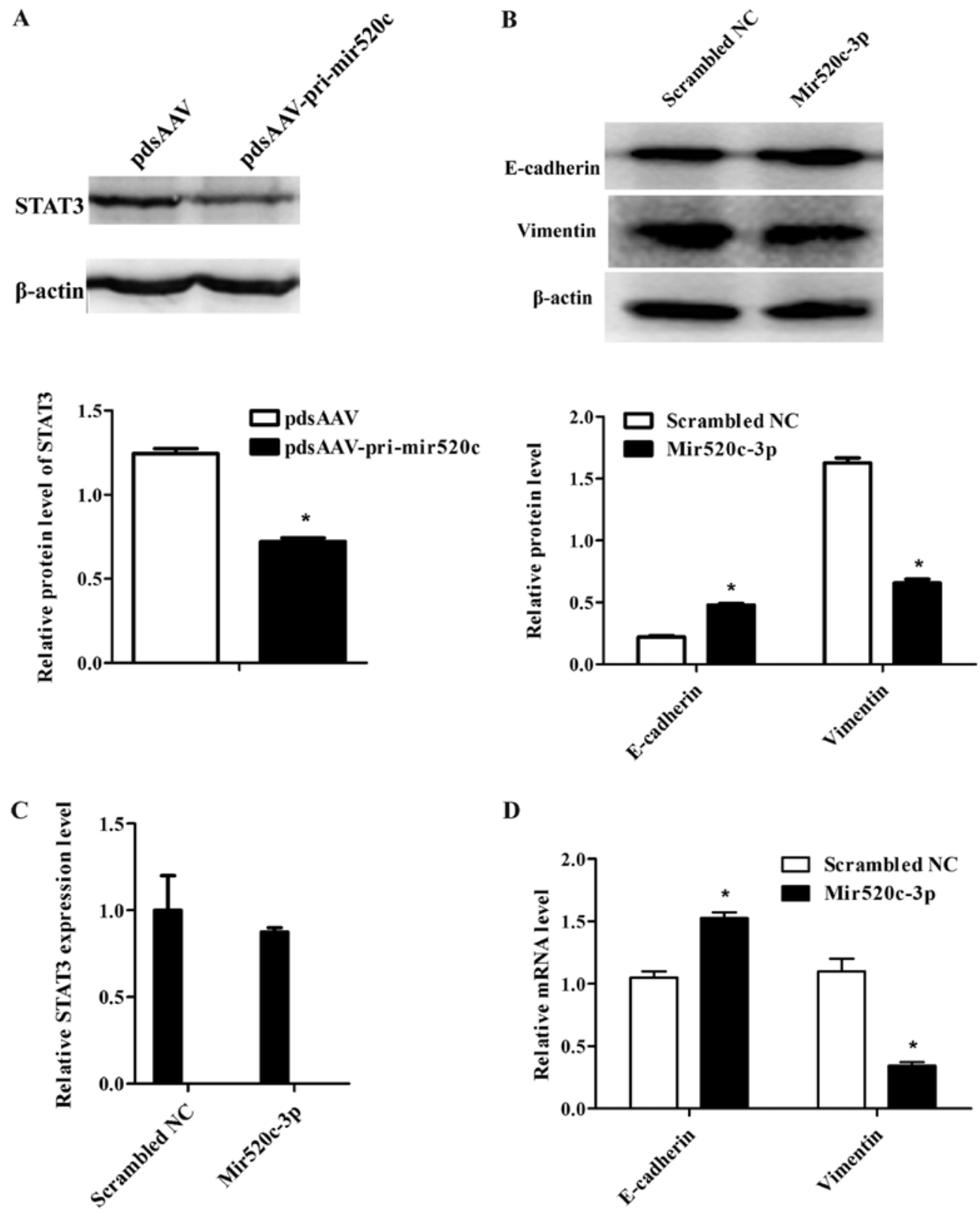

D

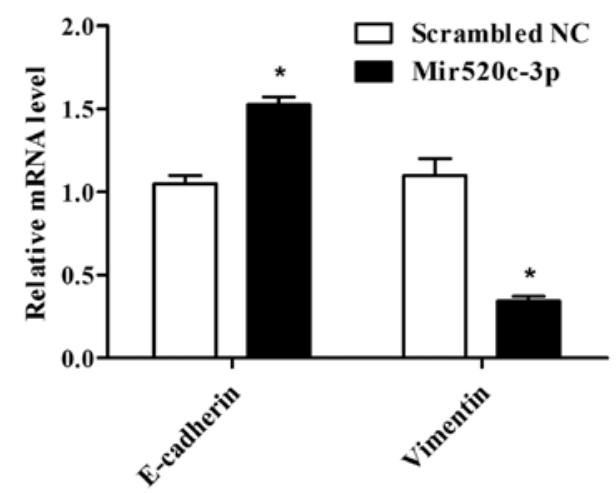

Figure 3. miR520c downregulates STAT3 in breast cancer cell line MCF-7. (A) Pri-miR520c was overexpressed in MCF-7, the STAT3 level was assessed by western blotting. (B) miR520c-3p mimics was overexpressed in MCF-7, the protein level of epithelial maker E-cadherin and mesenchymal marker Vimentin was detected by western blotting. (C) STAT3 was detected by Q-PCR after cells were transfected with scrambled/miR520c-3p mimics. (D) E-cadherin and Vimentin were detected by Q-PCR after cells were transfected with scrambled/miR520c-3p mimics. ${ }^{*} \mathrm{P}<0.05$.

could be increased (2-fold) when cells were transfected with miR520c inhibitor (Fig. 1E). Hence, as demonstrated, STAT3 3'UTR was directly regulated by miR520c.

The expression of STAT3 and miR520c in breast cancer cell lines. In 2014, Liu et al (25) reported that STAT3 was elevated in high grade breast cancers. We detected the mRNA and protein levels of STAT3 in three different grades of breast cancer cell lines: MCF-7, SK-BR-3 and MDA-MB-231. STAT3 was obviously upregulated (18-fold) in SK-BR-3 and (27-fold) in MDA-MB-231 compared to the low malignant MCF-7 (Fig. 2A). The higher metastatic cell line MDA-MB-231 expressed the highest mRNA and protein levels among the three cell lines. Activated form of STAT3-phosphorylated STAT3 (p-STAT3) of MCF-7 was significantly lower than the other two cell lines (Fig. 2C). Then we detected the expres- sion level of miR520c in three different progressions of breast cancer cells. Q-PCR results showed that the mRNA level of miR520c was decreased in MDA-MD-231, and MCF-7 expressed the highest level among the three breast cancer cell lines. Additionally, miR520c showed negative correlation with STAT3 expression level.

miR520c downregulates STAT3 in breast cancer cells. For further understanding of mechanism between miR520c and STAT3, we overexpressed miR520c mimics in MCF-7 cells. Western blotting result indicated that STAT3 protein level was significantly downregulated in miR520c overexressed group compared with the negative control group, it meant miR520c could inhibit STAT3 (Fig. 3A). Furthermore, we detected the mRNA level of STAT3, it was shown in the Q-PCR results 

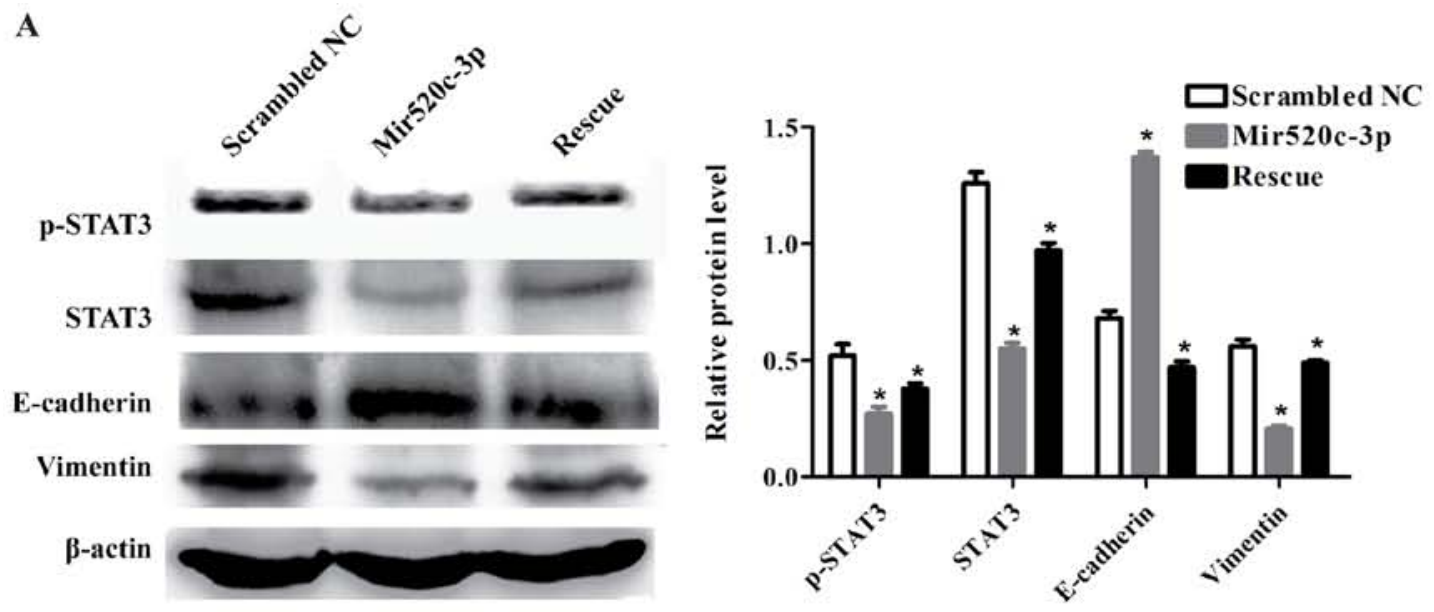

B
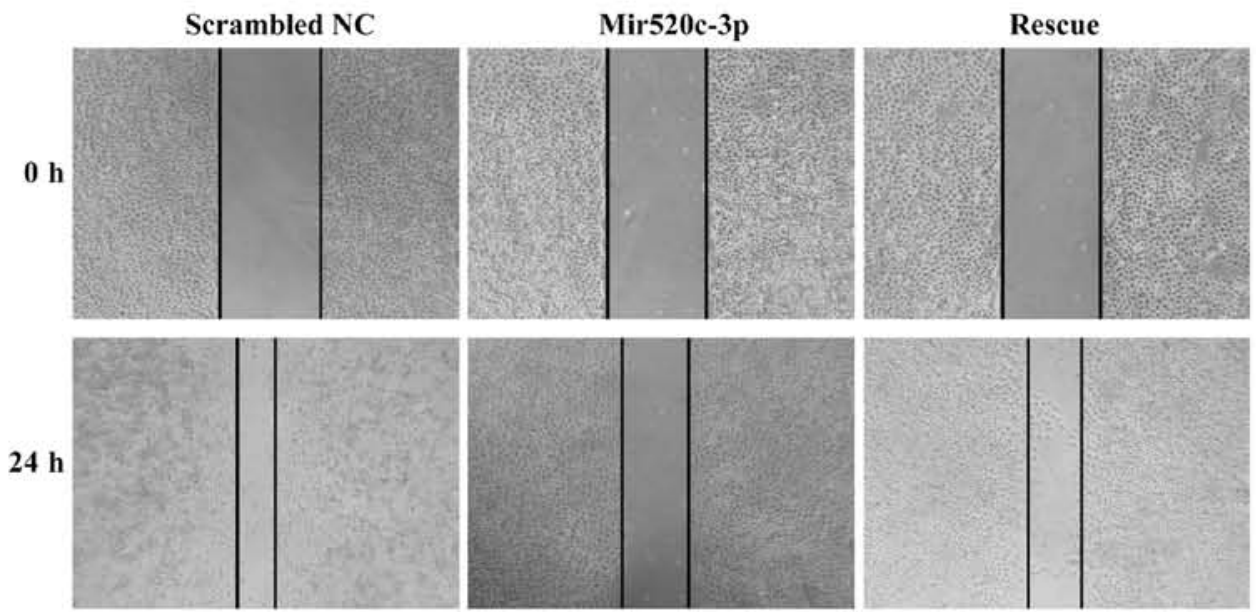

$(\times 40)$
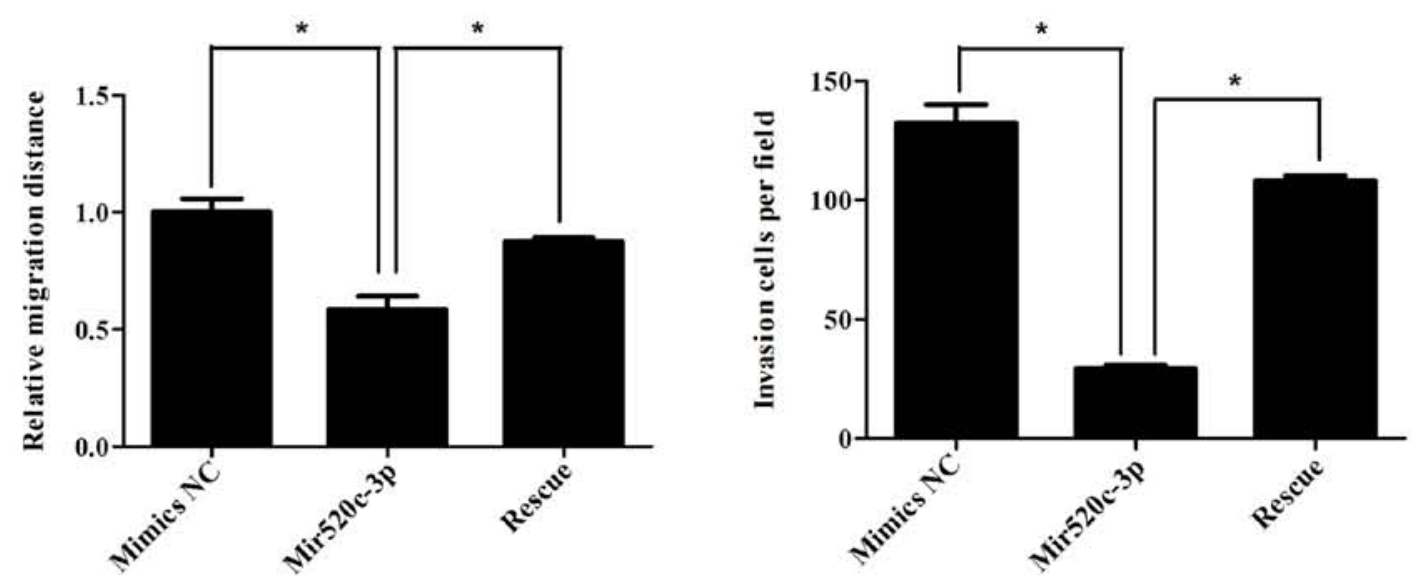

C
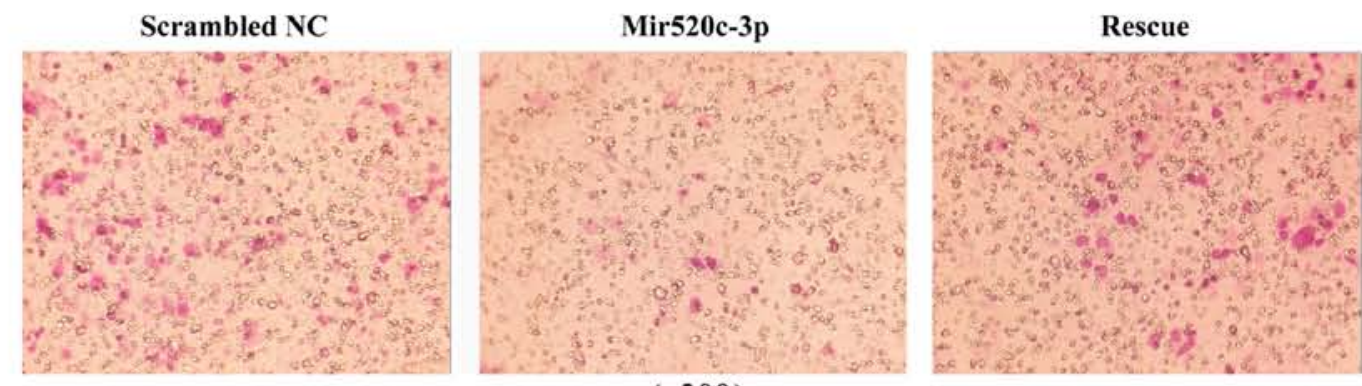

$(\times \mathbf{2 0 0})$

Figure 4. Overexpression of STAT3 in MCF-7 rescues the inhibition of EMT. (A) Cells were transfected with scrambled NC/miR520c-3p mimics/miR520c-3p mimics with STAT3 overexpression plasmid, p-STAT3, STAT3, Vimentin and E-cadherin were detected with western blotting after 48 h. (B) Scratch assay of the same groups with (A), the migration distance was recorded after $24 \mathrm{~h}$. Statistical analysis of relative migration distance and invasion cells per field. (C) Boyden invasion assay of the same groups with (A), $48 \mathrm{~h}$ after transfection, cells were seeded into chambers, and stained with crystal violet after $24 \mathrm{~h}$. ${ }^{*} \mathrm{P}<0.05$. 
that STAT3 was scarcely downregulated in miR520c overexpressed group. which indicated STAT3 was regulated by miR520c post-transcriptionally (Fig. 3C).

The breast cancer cells EMT progression is inhibited by miR520c targeting STAT3. EMT is one of the malignant cancer properties. There is remarkable change of tumor metastasis in epithelial to mesenchymal transition. When STAT3 was inhibited by miR520c, it demonstrated the EMT phenotype. Epthelial marker E-cadherin was significantly upregulated in miR520c overexpression group, and mesenchymal marker vimentin was downregulated at both protein and mRNA levels (Fig. 3B and D). These changes could be reversed by overexpression of STAT3 (Fig. 4A). Later on, we observed cell migration and invasion. It was shown in cell scratch assay, that miR520c inhibited cell motility and when we overexpressed STAT3 to rescue the transformation, we found the inhibition was partially abrogated (Fig. 4B). Similarly, miR520c suppressed cells invasion and migration, which could be rescued by STAT3 (Fig. 4C). These results demonstrated, miR520c suppressed STAT3, which led to inhibition of EMT.

\section{Discussion}

STAT3 is hyper-activated in cancers, approaches to inhibit STAT3 can be a key therapeutic task. In glioblastoma, BIS-mediated STAT3 stabilization can regulate stem cell-like phenotypes (26). In gastric cancer, suppressing the activation of STAT3 resensitized cells to chemotherapy (27). Inhibition of STAT3 has influence on cell cytotoxic and cytostatic effect, it also reinforces anticancer immunosurveillance to increase therapeutic efficacy and stem cell-like phenotype (28). In human Wilms tumor SK-NEP-1 cells, inhibiting STAT3 and CDDP reduced cell growth in vivo (29).

Besides, STAT3 can be downregulated by series of microRNAs. miR-23a, miR-27a and miR-24 coordinately exert JAK1/Stat3 cascade in human acute erythroid leukemia (30). miR-124 repressed proliferation in glioblastoma by targeting STAT3 (31). miR-125a promoted paclitaxel sensitivity via downregulation of STAT3 in cervical cancer (32).

It has been reported that mir520c also have functions in cancers. In isolated melanoma cancer stem cells, mir520c was identified to have relations with epithelial-to-mesenchymal transition and stem cell potential (33). miR-520c-3p suppresses diffuse large B cell lymphoma development by decreasing eIF4GII (34). Mir520c has dual functions in different types of cancers. It can act as a tumor suppressor, but it is also an oncomiR. The role of miR-520c in regulating activities of MMP2 and MMP9 was contrary (35). The mechanisms of miR520c inhibition in breast cancer carcinogenesis have not been fully illuminated. In our study, we selected miR520c as a potential regulator of STAT3. To make clear whether it promoted cancer progression, we screened the expression level of miR520c as well as STAT3, consistent with our expectations, with the rising of malignant grades, the mRNA level became lower and lower. Nevertheless, the post-transcriptional regulation of STAT3 in breast cancer may be multifactorial, including microRNAs. Afterwards, we validated miR520c bound with STAT3 3'UTR but not with the mutated one. Conversely, when we inhibited miR520c, the binding effect was attenuated. Furthermore, we verified overexpression of miR520c strongly decreased protein level of STAT3. It has been reported that STAT3 facilitates inflammation, proliferation, stem cell phenotype, metastasis. In our previous study, we detected apoptosis of breast cancer cells after transfected with miR520c, but no significant differences was observed. However, it has suppressing influence on EMT phenotypes. The epithelial marker E-cadherin was increased, on the contrary, mesenchymal marker vimentin was decreased. Cell motility and invasion ability were abrogated, but the rescue experiments partially recovered the repression effects, which means other mechanisms are needed to be regulated by miR520c.

Taken together, our data indicate STAT3 was a direct target of miR520c in breast cancer cell, and through this mechanism, the EMT process was repressed. It may lay a basis for breast cancer therapy and prognostic evaluation. Further work will be devoted to uncover the relationships between miR520c and STAT3 in cancer microenvironments and clinical cases.

\section{References}

1. De Martel C, Ferlay J, Franceschi S, Vignat J, Bray F, Forman D and Plummer M: Global burden of cancers attributable to infections in 2008: A review and synthetic analysis. Lancet Oncol 13: 607-615, 2012.

2. Parker JS, Mullins M, Cheang MC, Leung S, Voduc D, Vickery T, Davies S, Fauron C, He X, Hu Z, et al: Supervised risk predictor of breast cancer based on intrinsic subtypes. J Clin Oncol 27: 1160-1167, 2009.

3. Yaacoub K, Pedeux R, Tarte K and Guillaudeux T: Role of the tumor microenvironment in regulating apoptosis and cancer progression. Cancer Lett 378: 150-159, 2016.

4. Ma YF, Ren Y, Wu CJ, Zhao XH, Xu H, Wu DZ, Xu J, Zhang XL and Ji Y: Interleukin (IL)-24 transforms the tumor microenvironment and induces anticancer immunity in a murine model of colon cancer. Mol Immunol 75: 11-20, 2016.

5. Sweis RF, Spranger S, Bao R, Paner GP, Stadler WM, Steinberg GD and Gajewski TF: Molecular drivers of the non-T cell-inflamed tumor microenvironment in urothelial bladder cancer. Cancer Immunol Res 4: 563-568, 2016.

6. Fernandez-Garcia B, Eiró N, Miranda MA, Cid S, González LO, Domínguez F and Vizoso FJ: Prognostic significance of inflammatory factors expression by stroma from breast carcinomas. Carcinogenesis 37: 768-776, 2016.

7. Dieci MV, Griguolo G, Miglietta F and Guarneri V: The immune system and hormone-receptor positive breast cancer: Is it really a dead end? Cancer Treat Rev 46: 9-19, 2016.

8. Griesinger AM, Josephson RJ, Donson AM, Mulcahy Levy JM, Amani V, Birks DK, Hoffman LM, Furtek SL, Reigan P, Handler MH, et al: Interleukin-6/STAT3 pathway signaling drives an inflammatory phenotype in group A ependymoma. Cancer Immunol Res 3: 1165-1174, 2015.

9. Bromberg JF, Wrzeszczynska MH, Devgan G, Zhao Y, Pestell RG, Albanese C and Darnell JE Jr: Stat 3 as an oncogene. Cell 98: 295-303, 1999.

10. Yu H, Pardoll D and Jove R: STATs in cancer inflammation and immunity: A leading role for STAT3. Nat Rev Cancer 9: 798-809, 2009.

11. Garcia R and Jove R: Activation of STAT transcription factors in oncogenic tyrosine kinase signaling. J Biomed Sci 5: 79-85, 1998.

12. Meltzer PS: Cancer genomics: Small RNAs with big impacts. Nature 435: 745-746, 2005.

13. Benhamed M, Herbig U, Ye T, Dejean A and Bischof O: Senescence is an endogenous trigger for microRNA-directed transcriptional gene silencing in human cells. Nat Cell Biol 14: 266-275, 2012.

14. Filipowicz W, Bhattacharyya SN and Sonenberg N: Mechanisms of post-transcriptional regulation by microRNAs: Are the answers in sight? Nat Rev Genet 9: 102-114, 2008.

15. Kunej T, Godnic I, Ferdin J, Horvat S, Dovc P and Calin GA: Epigenetic regulation of microRNAs in cancer: An integrated review of literature. Mutat Res 717: 77-84, 2011. 
16. Calin GA, Sevignani C, Dumitru CD, Hyslop T, Noch E, Yendamuri S, Shimizu M, Rattan S, Bullrich F, Negrini M, et al: Human microRNA genes are frequently located at fragile sites and genomic regions involved in cancers. Proc Natl Acad Sci USA 101: 2999-3004, 2004.

17. Lu J, Getz G, Miska EA, Alvarez-Saavedra E, Lamb J, Peck D, Sweet-Cordero A, Ebert BL, Mak RH, Ferrando AA, et al: MicroRNA expression profiles classify human cancers. Nature 435: 834-838, 2005.

18. Ferdin J, Kunej T and Calin GA: Non-coding RNAs: Identification of cancer-associated microRNAs by gene profiling. Technol Cancer Res Treat 9: 123-138, 2010.

19. Fabbri M, Ivan M, Cimmino A, Negrini $M$ and Calin GA: Regulatory mechanisms of microRNAs involvement in cancer. Expert Opin Biol Ther 7: 1009-1019, 2007.

20. Yang YK, Xi WY,Xi RX,Li JY,Li Q and Gao YE: MicroRNA-494 promotes cervical cancer proliferation through the regulation of PTEN. Oncol Rep 33: 2393-2401, 2015.

21. Libório-Kimura TN, Jung HM and Chan EK: miR-494 represses HOXA10 expression and inhibits cell proliferation in oral cancer. Oral Oncol 51: 151-157, 2015.

22. Ward A, Shukla K, Balwierz A, Soons Z, König R, Sahin O and Wiemann S: MicroRNA-519a is a novel oncomir conferring tamoxifen resistance by targeting a network of tumour-suppressor genes in $\mathrm{ER}^{+}$breast cancer. J Pathol 233: 368-379, 2014

23. Rao X, Di Leva G, Li M, Fang F, Devlin C, Hartman-Frey C, Burow ME, Ivan M, Croce CM and Nephew KP: MicroRNA$221 / 222$ confers breast cancer fulvestrant resistance by regulating multiple signaling pathways. Oncogene 30: 1082-1097, 2011.

24. Keklikoglou I, Koerner C, Schmidt C, Zhang JD, Heckmann D, Shavinskaya A, Allgayer H, Gückel B, Fehm T, Schneeweiss A, et al: MicroRNA-520/373 family functions as a tumor suppressor in estrogen receptor negative breast cancer by targeting $\mathrm{NF}-\kappa \mathrm{B}$ and TGF- $\beta$ signaling pathways. Oncogene 31: 4150-4163, 2012.

25. Liu LY, Chang LY, Kuo WH, Hwa HL, Lin YS, Jeng MH, Roth DA, Chang KJ and Hsieh FJ: Correction to: Prognostic features of signal transducer and activator of transcription 3 in an $\mathrm{ER}(+)$ breast cancer model system. Cancer Inform 13: 125-129, 2014.

26. Im CN, Yun HH, Song B, Youn DY, Cui MN, Kim HS, Park GS and Lee JH: BIS-mediated STAT3 stabilization regulates glioblastoma stem cell-like phenotypes. Oncotarget 7: 35056-35070, 2016.
27. Yang H, Yamazaki T, Pietrocola F, Zhou H, Zitvogel L, Ma Y and Kroemer G: Improvement of immunogenic chemotherapy by STAT3 inhibition. OncoImmunology 5: e1078061, 2015.

28. Zhang JL, Liu XZ, Wang PY, Chen GW, Jiang Y, Qiao SK, Zhu J, Wang X, Pan YS and Liu YC: Targeting HCCR expression resensitizes gastric cancer cells to chemotherapy via down-regulating the activation of STAT3. Sci Rep 6: 24196, 2016.

29. Wang J, Zhang N, Qu H, You G, Yuan J, Chen C, Li W and Pan F: Inhibitory effect of STAT3 gene combined with CDDP on growth of human Wilms tumour SK-NEP-1 cells. Biosci Rep 36: e00342, 2016.

30. Su R, Dong L, Zou D, Zhao H, Ren Y, Li F, Yi P, Li L, Zhu Y, Ma Y, et al: microRNA-23a, -27a and -24 synergistically regulate JAK1/Stat 3 cascade and serve as novel therapeutic targets in human acute erythroid leukemia. Oncogene 35: 6001-6014, 2016.

31. Li W, Huang H, Su J, Ji X, Zhang X, Zhang Z and Wang H: miR-124 acts as a tumor suppressor in glioblastoma via the inhibition of signal transducer and activator of transcription 3. Mol Neurobiol: Mar 18, 2016 (Epub ahead of print).

32. Fan Z, Cui H, Yu H, Ji Q, Kang L, Han B, Wang J, Dong Q, Li Y, Yan Z, et al: MiR-125a promotes paclitaxel sensitivity in cervical cancer through altering STAT3 expression. Oncogenesis 5: e197, 2016.

33. Fomeshi MR, Ebrahimi M, Mowla SJ, Khosravani P, Firouzi J and Khayatzadeh $\mathrm{H}$ : Evaluation of the expressions pattern of miR-10b, 21, 200c, 373 and 520c to find the correlation between epithelial-to-mesenchymal transition and melanoma stem cell potential in isolated cancer stem cells. Cell Mol Biol Lett 20: 448-465, 2015.

34. Mazan-Mamczarz K, Zhao XF, Dai B, Steinhardt JJ, Peroutka RJ, Berk KL, Landon AL, Sadowska M, Zhang Y, Lehrmann E, et al: Down-regulation of eIF4GII by miR-520c-3p represses diffuse large B cell lymphoma development. PLoS Genet 10: e1004105, 2014.

35. Lu S, Zhu Q, Zhang Y, Song W, Wilson MJ and Liu P: DualFunctions of miR-373 and miR-520c by differently regulating the activities of MMP2 and MMP9. J Cell Physiol 230: 1862-1870, 2015. 